Original Research Article

\title{
Prospective in silico study of the feasibility and dosimetric advantages of MRI-guided dose adaptation for human papillomavirus positive oropharyngeal cancer patients compared with standard IMRT
}

\author{
Abdallah S.R. Mohamed ${ }^{\mathrm{a}, \mathrm{b}, \mathrm{c}, *}$, Houda Bahig ${ }^{\mathrm{a}, \mathrm{d}}$, Michalis Aristophanous ${ }^{\mathrm{e}}$, Pierre Blanchard ${ }^{\mathrm{f}}$, \\ Mona Kamal ${ }^{\mathrm{a}, \mathrm{g}}$, Yao Ding ${ }^{\mathrm{e}}$, Carlos E. Cardenas ${ }^{\mathrm{e}}$, Kristy K. Brock ${ }^{\mathrm{h}}$, Stephen Y. Lai ${ }^{\mathrm{i}}$, \\ Katherine A. Hutcheson ${ }^{\mathrm{i}}$, Jack Phan ${ }^{\mathrm{a}}$, Jihong Wang ${ }^{\mathrm{e}}$, Geoffrey Ibbott ${ }^{\mathrm{e}}$, Refaat E. Gabr ${ }^{\mathrm{j}}$, \\ Ponnada A. Narayana ${ }^{j}$, Adam S. Garden ${ }^{a}$, David I. Rosenthal ${ }^{\text {a }, ~ G . ~ B r a n d o n ~ G u n n ~}{ }^{\mathrm{a}}$, \\ Clifton D. Fuller ${ }^{a}$,*, MD Anderson MRLinac Development Working Group \\ a Department of Radiation Oncology, The University of Texas MD Anderson Cancer Center, Houston, TX, USA \\ ${ }^{\mathrm{b}}$ Department of Clinical Oncology and Nuclear Medicine, Faculty of Medicine, University of Alexandria, Alexandria, Egypt \\ ${ }^{\mathrm{c}}$ MD Anderson Cancer Center/UT Health Graduate School of Biomedical Sciences, Houston, TX, USA \\ d Department of Radiation Oncology, Centre hospitalier de l'Université de Montréal (CHUM), Canada \\ e Department of Radiation Physics, The University of Texas MD Anderson Cancer Center, Houston, TX, USA \\ ${ }_{\mathrm{f}}^{\mathrm{f}}$ Department of Radiation Oncology, Institut de Cancérologie Gustave Roussy, Paris, France \\ ${ }^{g}$ Department of Clinical Oncology and Nuclear Medicine, Faculty of Medicine, University of Ain Shams, Cairo, Egypt

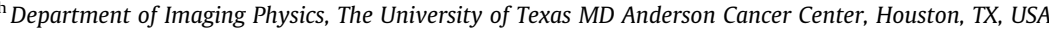 \\ ${ }^{\mathrm{i}}$ Department of Head and Neck Surgery, The University of Texas MD Anderson Cancer Center, Houston, TX, USA \\ ${ }^{j}$ Department of Diagnostic E' Interventional Imaging, The University of Texas Health Science Center at Houston, Houston, TX, USA
}

\section{A R T I C L E I N F O}

\section{Article history:}

Received 15 February 2018

Revised 17 April 2018

Accepted 30 April 2018

Available online 5 May 2018

\section{Keywords:}

MRI-guided radiotherapy

Dose adaptation

IMRT

Human papillomavirus positive

oropharyngeal cancer

Head and neck cancer

Dysphagia

Normal tissue complication probability

\begin{abstract}
A B S T R A C T
Purpose: We aim to determine the feasibility and dosimetric benefits of a novel MRI-guided IMRT doseadaption strategy for human papillomavirus positive ( $\mathrm{HPV}+)$ oropharyngeal squamous cell carcinoma (OPC).

Materials/methods: Patients with locally advanced HPV+ OPC underwent pre-treatment and in-treatment MRIs every two weeks using RT immobilization setup. For each patient, two IMRT plans were created (i.e. standard and adaptive). The prescription dose for the standard plans was $2.12 \mathrm{~Gy} / \mathrm{fx}$ for 33 fractions to the

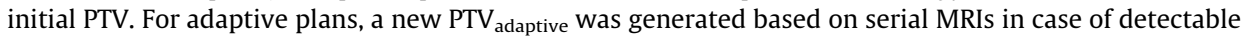
tumor shrinkage. Prescription dose to PTV adaptive was $2.12 \mathrm{~Gy} / \mathrm{fx}$ to allow for maximum dose to the residual disease. Any previously involved volumes received minimally a floor dose of $50.16 \mathrm{~Gy}$. Uninvolved elective nodal volumes were prescribed $50.16 \mathrm{~Gy}$ in $1.52 \mathrm{~Gy} / \mathrm{fx}$. Dosimetric parameters of organs at risk (OARs) were recorded for standard vs. adaptive plans. Normal tissue complication probability (NTCP) for toxicity endpoints was calculated using literature-derived multivariate logistic regression models.

Results: Five patients were included in this pilot study, 3 men and 2 women. Median age was 58 years (range 45-69). Three tumors originated at the tonsillar fossa and two at the base of tongue. The average dose to $95 \%$ of initial PTV volume was $70.7 \mathrm{~Gy}$ (SD,0.3) for standard plans vs. 58.5 Gy (SD,2.0) for adaptive plans. The majority of OARs showed decrease in dosimetric parameters using adaptive plans vs. standard plans, particularly swallowing related structures. The average reduction in the probability of developing dysphagia $\geq$ grade2, feeding tube persistence at 6-month post-treatment and hypothyroidism at 1-year post-treatment was $11 \%, 4 \%$, and $5 \%$, respectively. The probability of xerostomia at 6 -month was only reduced by $1 \%$ for adaptive plans vs. standard IMRT.

Conclusion: These in silico results showed that the proposed MRI-guided adaptive approach is technically feasible and advantageous in reducing dose to OARs, especially swallowing musculature.

(c) 2018 The Authors. Published by Elsevier B.V. on behalf of European Society for Radiotherapy and

Oncology. This is an open access article under the CC BY-NC-ND license (http://creativecommons.org/
\end{abstract} licenses/by-nc-nd/4.0/).

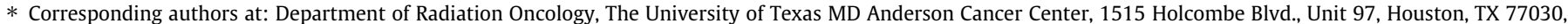
USA.

E-mail addresses: asmohamed@mdanderson.org (A.S.R. Mohamed), cdfuller@mdanderson.org (C.D. Fuller).
} 


\section{Introduction}

Human-papilloma virus positive (HPV+) oropharyngeal cancer (OPC) is epidemic in the United States, with an estimated 20,000 new cases annually, and rising incidence projected in the coming decades [1]. HPV+ cancers are sensitive to radiotherapy but despite excellent survival outcomes and the introduction of intensity modulated radiotherapy, current regimens continue to be associated with toxicity to adjacent normal tissue [2-8]. This leaves comparatively young survivors with potentially quality-oflife altering, permanent radiation sequelae that can persist for decades of survivorship, and limit future compensatory functionality in the face of new challenges [9-12]. To address this issue, it is necessary to find the optimal therapeutic window of HPV+ OPC, where dose to organs at risk (OARs) can be reduced while tumoricidal doses to active tumor volumes can be achieved. However, safely achieving this target by anatomically adapting the dose to follow serially shrinking tumor volumes during the 6-7 week radiation therapy course is currently impossible using CT without repeated use of exogenous contrast. In addition, existing functional imaging biomarkers, such as radiolabeled positron emission tomography (PET) tracers cannot be safely repeated iteratively during treatment. Therefore, the ability to image tumors during therapy to adapt radiation fields for responding tumors, reducing OAR dose and subsequent toxicity, is currently an unmet need.

Adaptive radiotherapy strategies have been previously implemented at our facility $[13,14]$. Schwartz et al. performed adaptive replanning mid-therapy for head and neck cancer patients, using daily computed tomography (CT)-on-rails imageguidance. The lack of contrast delivery for the CT-on-rails impeded the accurate visualization of tumor changes during treatment, and thus did not allow for reduction of clinical target volumes as tumor shrank, but instead accounted for weight-loss and normal tissue deformation [13,14]. Magnetic resonance imaging (MRI) provides superior tumor/soft tissue contrast [15]. In a recent study by our group [16], 31 patients with locally advanced HPV+ OPC were examined for mid-treatment response as assessed by MRI. The study showed that approximately $50 \%$ of patients had complete resolution of clinical and radiographically primary disease at mid-therapy. Using serial MRI-guided dose adaptation in this cohort of patients would allow selective, patient-specific precise dose-reduction, such that patients with brisk radiation response would have commensurate dose reduction, while comparatively radiation resistant tumor subvolumes would be ensured a tumoricidal dose. Using serial in-treatment MRI without exogenous/IV contrast, we can potentially track tumor shrinkage during treatment, conceivably de-escalating OARs doses to reduce side effects without sacrificing locoregional control and survival.

In this dosimetric study, we propose a novel MRI-guided IMRT dose-adaption strategy for $\mathrm{HPV}+\mathrm{OPC}$, whereby dose to gross disease is reduced on an "as needed" basis, such that responders could achieve substantive dose reduction to adjacent normal tissue at levels not observed with standard radiotherapy, while non-responsive disease would not be a priori de-escalated. This represents a truly "personalized" therapy, as, rather than assigning dose a priori, the cumulative dose received by each patient would be predicated on imaging response. To this end, we aim to determine the feasibility and dosimetric benefits of this MRI-based dose-adaption strategy for HPV+ OPC patients using serial in-treatment MRIs acquired in radiation treatment positioning and immobilization setup.

\section{Materials and methods}

\section{Patients}

Patients in the current study were prospectively enrolled under an Institutional Review Board (IRB)-approved imaging protocol (PA14-0582) after signing a study-specific informed consent form. Patients were scanned between July 2015 and June 2016. Inclusion criteria were age older than 18 years; histologically proven $\mathrm{P} 16+$ oropharyngeal squamous cell carcinoma; eligibility for definitive IMRT; intact primary tumor; Stage III, IVa, or IVb disease as defined by American Joint Committee on Cancer (AJCC) 7th edition cancer staging criteria; ECOG performance status of $0-2$; no administration of induction chemotherapy before radiotherapy; and no contraindications to MR imaging.

\section{MRI protocol}

Serial MRI simulation images were acquired at baseline (within one week prior to first radiation fraction), and every two weeks during the IMRT course (i.e. at weeks 2, 4, and 6). Patients were dispositioned to receive a custom-fitted oral stent and an immobilization mask same to that used for radiotherapy treatment planning prior to receiving their study MRIs. The stent was made by the dental oncology team to hold the tongue and the remainder of the oral cavity in place. The thermoplastic mesh mask, for the head and neck region, was made during the simulation phase to immobilize the head, neck, and shoulders of the patient in a reproducible way. We previously detailed the positioning and immobilization setup for our MRI-simulation process in a separate publication [17].

Patients' images were acquired using a 3.0 T MR scanner (Ingenia, Philips Healthcare, Best, The Netherlands) with two SENSE Flex-M coils laterally and SENSE spine coil posteriorly. MRI sequences included axial T2 weighted image (repetition time/echo time $=8755 / 100 \mathrm{~ms}$, echo train length $=15$, field of view $=25.6 \mathrm{~cm}$, spatial resolution $=0.5 \times 0.5 \times 2.5 \mathrm{~mm}^{3}$, number of signal averages $=2$, pixel bandwidth $=184 \mathrm{~Hz}$, number of slices $=90$ ). Geometrical scan parameters were prescribed for a standardized spatial region encompassing the vertex cranially to the cricoid cartilage caudally for all scans.

\section{CT simulation}

Standard simulation CTs were acquired for each patient at baseline prior to treatment, followed by serial simulation CT imaging for adaptive replanning at the same time points of MRsimulation (i.e. at weeks 2,4 , and 6) using identical positioning and immobilization setup (see schema of protocol, Fig. 1).

\section{Target volumes and dose specification}

Target volumes were delineated by the study PI (CDF) and were peer reviewed by MD Anderson's Radiation Oncology Head-andNeck Planning and Development Clinic. The process of peerreview of segmented contours was explained in details in a prior report by our group [18]. In brief, the process entails comprehensive review of a patient's history, pathology, diagnostic imaging, and discussion of the planned treatment. All patients undergo physical examination (PE) including video-camera nasopharyngolaryngoscopy and bimanual palpation performed by a team of head-and-neck radiation oncology sub-specialists. The proposed segmentations were reviewed slice-by-slice for gross tumor volume (GTV), clinical target volume (CTV) and OAR segmentation, as well as dose-volume specifications. By this manner, intra- and 


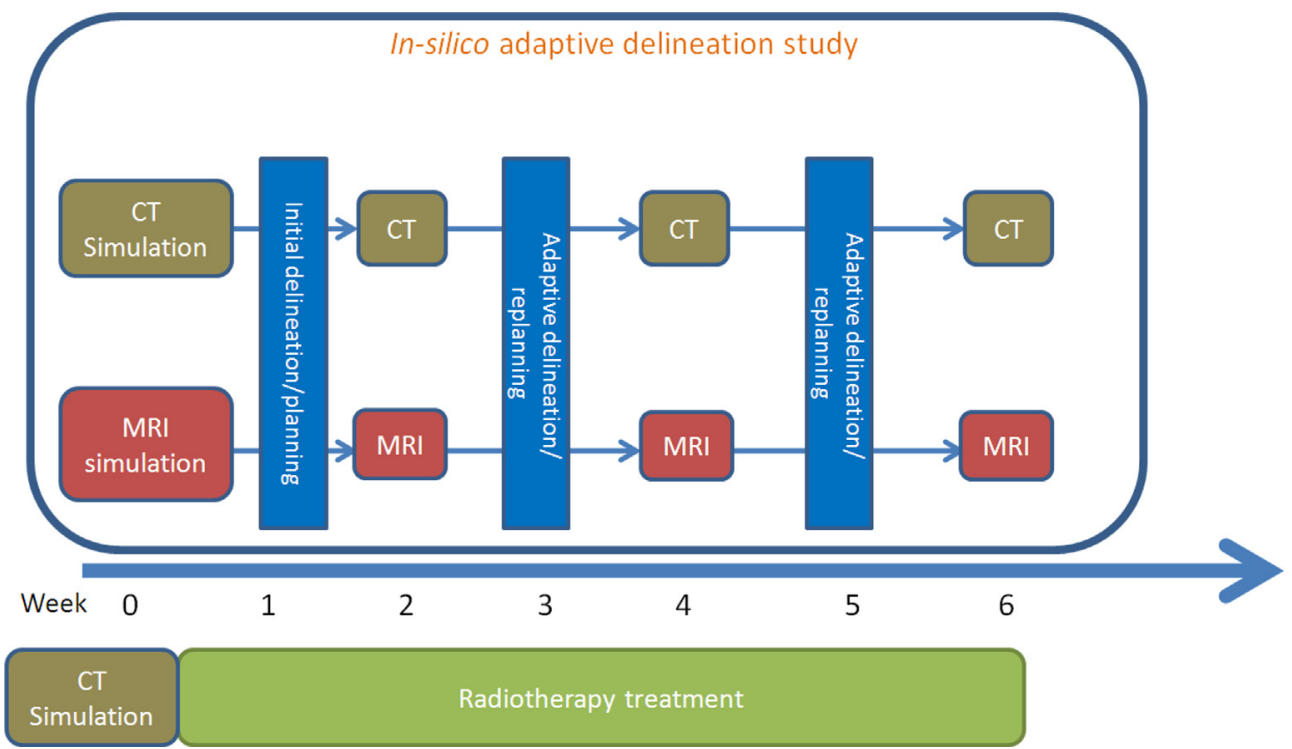

Fig. 1. Schema of in silico adaptive planning protocol.

inter-observer variability in segmentation are minimized because of the utilization of multi-observers agreement contours rather than single-observer contours.

The initial gross tumor volume (e.g. GTV_p initial $_{\text {for primary dis- }}$ ease and GTV_n $\mathrm{n}_{\text {initial }}$ for nodal disease) was manually segmented using T2-weighted MR images at baseline then propagated to the co-registered simulation CT acquired at the same day. The initial clinical target volume $\left(\mathrm{CTV}_{\text {initial }}\right)$ was defined as the $\mathrm{GTV}_{\text {initial }}$ plus $5 \mathrm{~mm}$ expansion, trimmed from uninvolved bone, muscle, skin or mucosal surfaces; to incorporate high-risk subclinical disease.

For each patient, two IMRT plans were created: a standard and an adaptive treatment plan. The prescription dose for the standard plans was $2.12 \mathrm{~Gy} / \mathrm{fx}$ for 33 fractions to the $\mathrm{PTV}_{\text {initial }}\left(\mathrm{CTV}_{\text {initial }}\right.$ $+3 \mathrm{~mm}$ ). For adaptive plans, a new GTV adaptive was segmented on serial MRIs using T2-weighted MR images at time points showing a detectable shrinkage of the $\mathrm{GTV}_{\text {initial. }}$ Subsequently, a new
$\mathrm{CTV}_{\text {adaptive }}$ was generated to cover the $\mathrm{GTV}_{\text {adaptive }}$ propagated from MRI to the corresponding same day CT with additional $5 \mathrm{~mm}$ margin. Detectable shrinkage was defined as any GTV $_{\text {initial }}$ reduction of more than $2 \mathrm{~mm}$ in the reference plane (largest cross sectional distance axially on the pretherapy imaging).

The prescription dose to PTV $_{\text {adaptive }}\left(\mathrm{CTV}_{\text {adaptive }}+3 \mathrm{~mm}\right)$ was $2.12 \mathrm{~Gy} / \mathrm{fx}$ to allow delivery of maximum dose to the residual disease, resulting in a cumulative dose, should disease persist through therapy, of up to $70 \mathrm{~Gy}$. Prescription dose for any previously involved volumes was $1.52 \mathrm{~Gy} / \mathrm{fx}$ to ensure a minimum "floor" dose of 50.16 Gy to any region ever deemed to have been directly involved with tumor. All uninvolved upper-neck elective nodal volumes outside the $\mathrm{CTV}_{\text {initial }} / \mathrm{CTV}_{\text {adaptive }}$ were encompassed in the

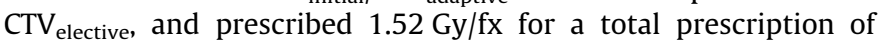
$50.16 \mathrm{~Gy} / 33$ fractions. Fig. 2 illustrates the workflow for adaptive vs. standard plans.

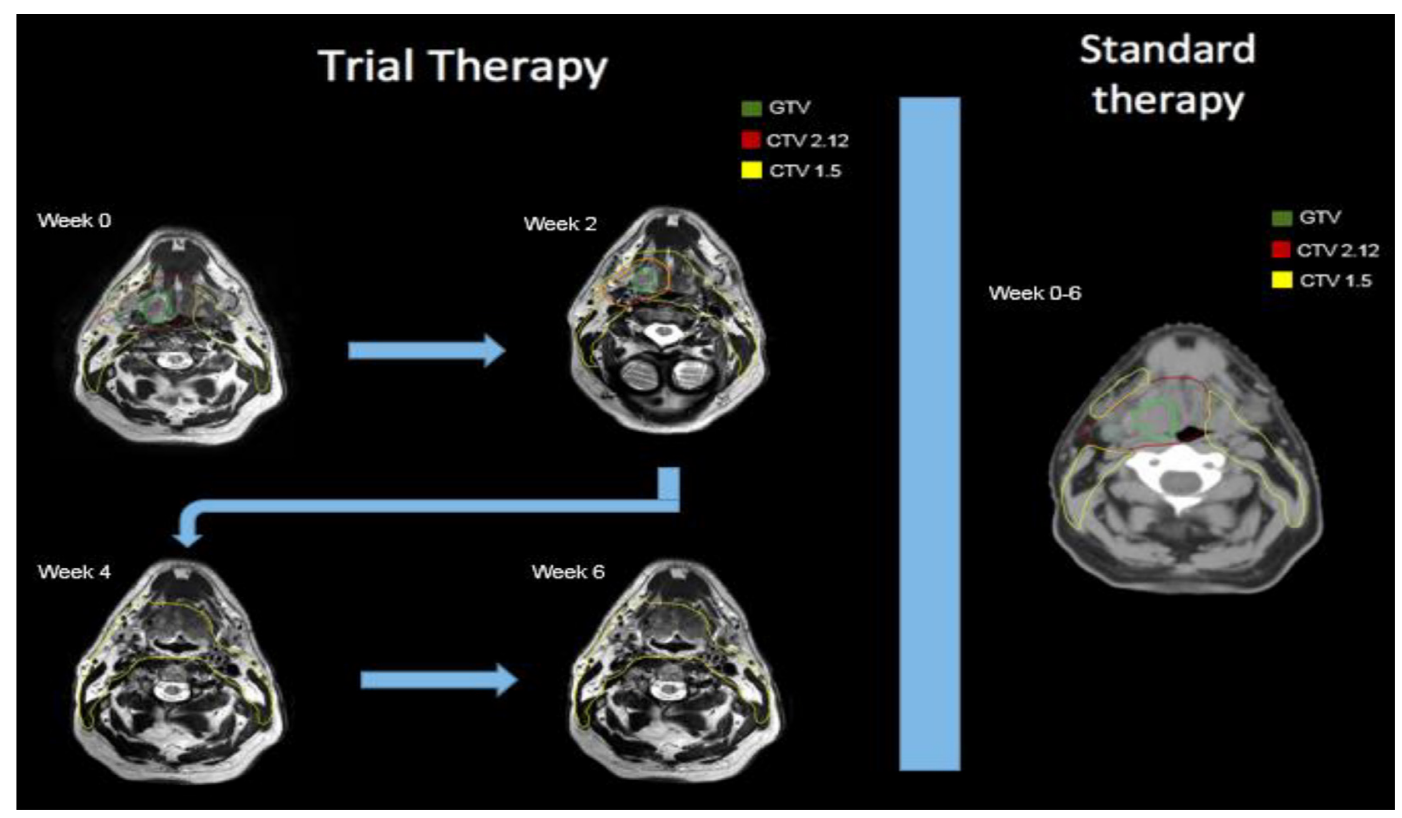

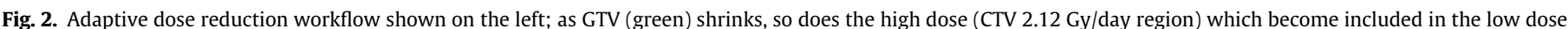

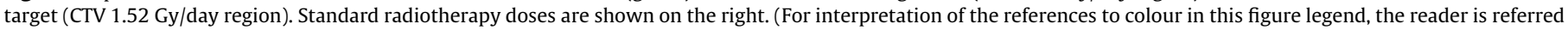
to the web version of this article.) 
Table 1

Patient demographic, disease, and treatment characteristics.

\begin{tabular}{|c|c|c|c|c|c|c|c|c|}
\hline Patient & Origin & Gender & Age (years) & Smoking history & T stage & $\mathrm{N}$ stage & AJCC stage & Concurrent chemotherapy \\
\hline 1 & Tonsil & Female & 45 & Never & $\mathrm{T} 2$ & $\mathrm{~N} 2 \mathrm{c}$ & IVA & Weekly Cisplatin \\
\hline 2 & Base of Tongue & Male & 60 & Never & $\mathrm{T} 2$ & N1 & III & No chemotherapy \\
\hline 3 & Tonsil & Female & 69 & Former & $\mathrm{T} 2$ & $\mathrm{~N} 2 \mathrm{~b}$ & IVA & Weekly Cetuximab \\
\hline 4 & Tonsil & Male & 51 & Never & $\mathrm{T} 2$ & $\mathrm{~N} 2 \mathrm{c}$ & IVA & Weekly Cisplatin \\
\hline 5 & Base of Tongue & Male & 58 & Never & $\mathrm{T} 2$ & $\mathrm{~N} 2 \mathrm{~b}$ & IVA & Weekly Cetuximab \\
\hline
\end{tabular}

\section{OAR segmentation}

Organs at risk (OARs) were auto-segmented on simulation CTs at baseline and at weeks 2, 4, and 6 using a previously validated atlas-based auto-segmentation software program ADMIRE v1.13 (Elekta AB, Stockholm, Sweden). This was followed by review and correction of the contours when needed by an experienced radiation oncologist (ASRM). The following OARs were included: spinal cord; brain stem; bilateral parotid and submandibular glands; thyroid gland; larynx; oral cavity; brachial plexus; superior, middle, and inferior pharyngeal constrictors; medial and lateral pterygoid muscles; masseter; sternocleidomastoid; intrinsic and extrinsic tongue muscles; hard palate; and soft palate.

\section{Radiation planning}

All plans were optimized to full dose (70 Gy or $50.16 \mathrm{~Gy}$ if no residual disease was present) to keep the total dose to each OAR below the tolerance limit for every adaptive plan while maintaining at least $99 \%$ coverage to the PTV with a hot spot less than $110 \%$ to ensure that no normal tissue limit would be reached for a specific organ before the end of treatment. Once the plan was finalized, the number of fractions was adjusted to the number that would be delivered for the next adaptive phase. Dose accumulation was performed at the end of each adaptive phase to ensure target volumes met prescription dose and OARs met dose constraints.

Planning was performed with Pinnacle3 v.9.10 (Philips Medical Systems, Fitchburg, WI). All patients were planned with volumetric modulated arc therapy. For bilateral neck irradiation, two 360 degree arcs were utilized, while for cases of unilateral neck irradiation, two half arcs were used. The duration of the MRI-simulation was one hour and the duration of segmentation and replanning was four hours per patient.

\section{Statistical analysis}

Three dimensional volumetric changes of GTV_p and GTV_n were recorded at all time points. Dosimetric parameters of target volumes and OARs were recorded for standard vs. adaptive plans for each patient. Subsequently, normal tissue complication probability (NTCP) for toxicity endpoints was calculated using literaturederived multivariate logistic regression models [19-22]. The toxicity endpoints examined were: 1) persistence of feeding tube 6 months after treatment [19], 2) grade $\geq 2$ dysphagia 6 months after treatment [20], 3) hypothyroidism 12 months after treatment [21], and 4) xerostomia 6 months after treatment [22]. The rationale for NTCP model selection was detailed in a previous publication by our group [23]. All statistical analyses were performed using statistical software (JMP Pro version 11, SAS Institute, Cary, NC).

\section{Results}

Five patients were included in this pilot study; 3 men and 2 women. Median age was 58 years (range 45-69). Three tumors originated at the tonsillar fossa and two at the base of tongue.
Patient demographic, disease, and treatment characteristics are summarized in Table 1.

The average decrease in GTV_p volume at weeks 2, 4, and 6 was $44 \%, 90 \%$, and $100 \%$, respectively. The GTV_n volume shrinkage, however, had a relatively slower pace with average decrease in GTV_n volume at weeks 2,4 , and 6 of $25 \%, 60 \%$, and $80 \%$, respectively. These significant shrinkage qualified all patients for adaptive plans at weeks 2, 4, and 6. The course of target volume response is presented graphically in Fig. 3 for all patients included in the analysis.

Results demonstrated that the vast majority of OARs showed a decrease in dosimetric parameters when adaptive plans were used compared with standard plans, particularly for swallowing related structures, as illustrated in Table 2. Regarding target volumes, the

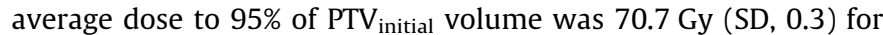
standard plans versus $58.5 \mathrm{~Gy}$ (SD, 2.0) for adaptive plans. Details of dose parameters for target volumes are presented in Supplementary Table 1.

Using NTCP models, the average reduction of the probability of developing dysphagia $\geq$ grade 2 and feeding tube persistence at 6 month post-treatment using adaptive strategy was $11 \%$ ( $37 \%$ vs $26 \%$, odds ratio $(\mathrm{OR})=0.6,95 \% \mathrm{CI} 0.2-1.5)$ and $4 \%(10 \%$ vs $6 \%$, OR $=0.5,95 \%$ CI $0.1-3)$, respectively as depicted in Fig. 4 .

The probability of developing hypothyroidism at 1-year posttreatment was also reduced by average $5 \%(41 \%$ vs $36 \%$, OR $=0.8$, 95\% CI 0.3-2) while the probability of xerostomia at 6-month was only reduced by average $1 \%$ for adaptive plans compared with standard IMRT (35\% vs 34\%, OR = 0.95, 95\% CI 0.4-2.5).

\section{Discussion}

In this study, we report the feasibility of an MRI-guided IMRT dose-adaption workflow for HPV+ OPC. To our knowledge, this is the first study reporting on the dosimetric advantage of MRIbased adaptive radiation de-intensification in head and neck cancers. The proposed approach was associated with an average reduction in the dose to the PTV of $12 \mathrm{~Gy}$. Adaptive replanning was associated with reduction of dose to the OARs, in particular to the swallowing musculature, which translated into a reduction of the odds of dysphagia $\geq$ grade 2 , feeding tube persistence at 6 months, and hypothyroidism at 1 -year post-treatment.

$\mathrm{HPV}+\mathrm{OPC}$ has been shown to be a favorable subtype of head and neck cancer with improved prognosis compared to non-HPV + OPC $[24,25]$. The distinctive epidemiologic, clinical and molecular characteristics [26] of HPV+ OPC are now reflected in the new cancer staging proposed in the American Joint Committee on Cancer 8th edition [27]. Given the excellent outcomes of HPV+ OPC, it is increasingly considered that many patients with HPV+ OPC may be over-treated with current standard chemoradiation. It is, in fact, recognized that current standard treatment is associated with high rates of toxicities that were shown to adversely impact patients' health-related quality of life [28]. Given the high probability of long-term survival and typical young age of patients with HPV+ OPC, treatment de-intensification aiming at reducing long-term toxicities and improving survivorship has become a central concern in the management of these patients [29]. To this end, multi- 


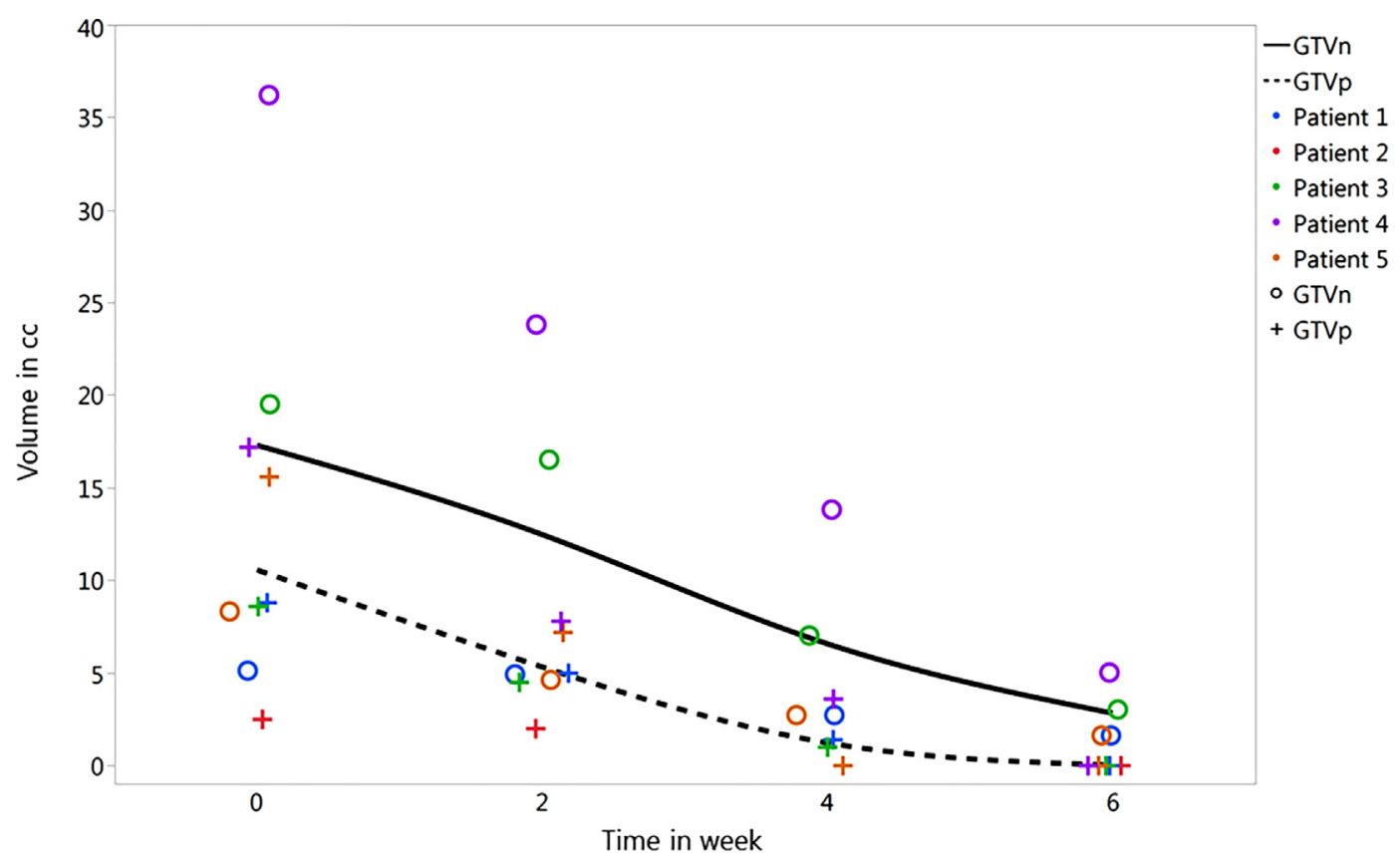

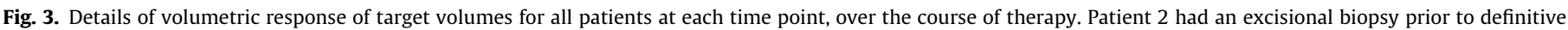
IMRT and, therefore, had no GTVn at radiation start.

ple clinical trials are currently on-going to assess various treatment de-escalation strategies in this group [30,31].

The overall goal of all treatment de-intensification strategies is to maintain excellent cancer outcomes while reducing morbidity. Current evaluated strategies include the use of targeted therapies versus systemic chemotherapy [30], reduced radiation dose based on response to induction chemotherapy response [32-34], or modulation of radiation dose in the context of chemoradiation [35,36]. Proton therapy may be also an alternative way to reduce normal tissue toxicity and is currently investigated in a randomized trial
(NCT01893307) comparing IMPT versus standard IMRT. A recent study by Blanchard et al. demonstrated the validity of a set of NTCP models for head and neck cancer patients treated with proton therapy. However, improvement in model performance remains to be required for better selection of patients for proton therapy [37]. Furthermore, minimally invasive surgery such as trans-oral robotic surgery (TORS) has been also introduced as an alternative approach to avoid radiation toxicity with equivalent oncologic outcomes [38]. An ongoing randomized clinical trial (NCT02984410) is currently assessing the patient-reported swallowing function over

Table 2

Dosimetric criteria of organs at risk using standard vs adaptive plans.

\begin{tabular}{|c|c|c|c|c|}
\hline Organ at risk (OAR) & $\begin{array}{l}\text { Mean dose Standard IMRT in } \\
\text { Gy }\end{array}$ & $\begin{array}{l}\text { Standard Deviation Standard } \\
\text { IMRT Gy }\end{array}$ & $\begin{array}{l}\text { Mean dose Adaptive IMRT in } \\
\text { Gy }\end{array}$ & $\begin{array}{l}\text { Standard Deviation Adaptive } \\
\text { IMRT Gy }\end{array}$ \\
\hline Supraglottic larynx & 52.7 & 10.7 & 45.8 & 10.4 \\
\hline Glottic larynx & 33.8 & 21.7 & 31.0 & 18.9 \\
\hline $\begin{array}{l}\text { Superior pharyngeal } \\
\text { constrictor }\end{array}$ & 62.8 & 6.7 & 58.1 & 5.0 \\
\hline Middle pharyngeal constrictor & 51.6 & 16.4 & 48.4 & 12.5 \\
\hline Inferior pharyngeal constrictor & 34.7 & 23.3 & 32.0 & 18.6 \\
\hline Cricopharyngeus muscle & 30.0 & 19.0 & 27.5 & 17.5 \\
\hline Mylo/geniohyoid muscle & 37.8 & 10.5 & 33.4 & 11.2 \\
\hline Intrinsic tongue muscles & 44.7 & 14.5 & 40.1 & 12.9 \\
\hline Genioglossus muscle & 51.8 & 13.5 & 47.4 & 11.0 \\
\hline Oral cavity & 42.1 & 11.3 & 38.0 & 10.8 \\
\hline Soft palate & 55.0 & 10.7 & 49.2 & 10.6 \\
\hline $\begin{array}{l}\text { Ipsilateral ant. Digastric } \\
\text { muscle }\end{array}$ & 44.4 & 6.9 & 40.6 & 7.1 \\
\hline $\begin{array}{l}\text { Contralateral ant. Digastric } \\
\text { muscle }\end{array}$ & 29.9 & 10.2 & 26.0 & 12.7 \\
\hline Ipsilateral parotid gland & 30.2 & 11.3 & 26.9 & 8.3 \\
\hline Contralateral parotid gland & 17.4 & 8.6 & 16.5 & 8.4 \\
\hline $\begin{array}{l}\text { Ipsilateral submandibular } \\
\text { gland }\end{array}$ & 69.7 & 3.6 & 65.0 & 6.0 \\
\hline $\begin{array}{l}\text { Contralateral submandibular } \\
\text { gland }\end{array}$ & 40.9 & 20.3 & 39.9 & 19.6 \\
\hline Esophagus & 19.2 & 12.3 & 16.8 & 9.9 \\
\hline Brain Stem & 10.8 & 1.9 & 8.3 & 2.7 \\
\hline Spinal cord & 21.8 & 6.5 & 21.2 & 8.0 \\
\hline Thyroid gland & 36.1 & 23.2 & 32.8 & 20.7 \\
\hline
\end{tabular}




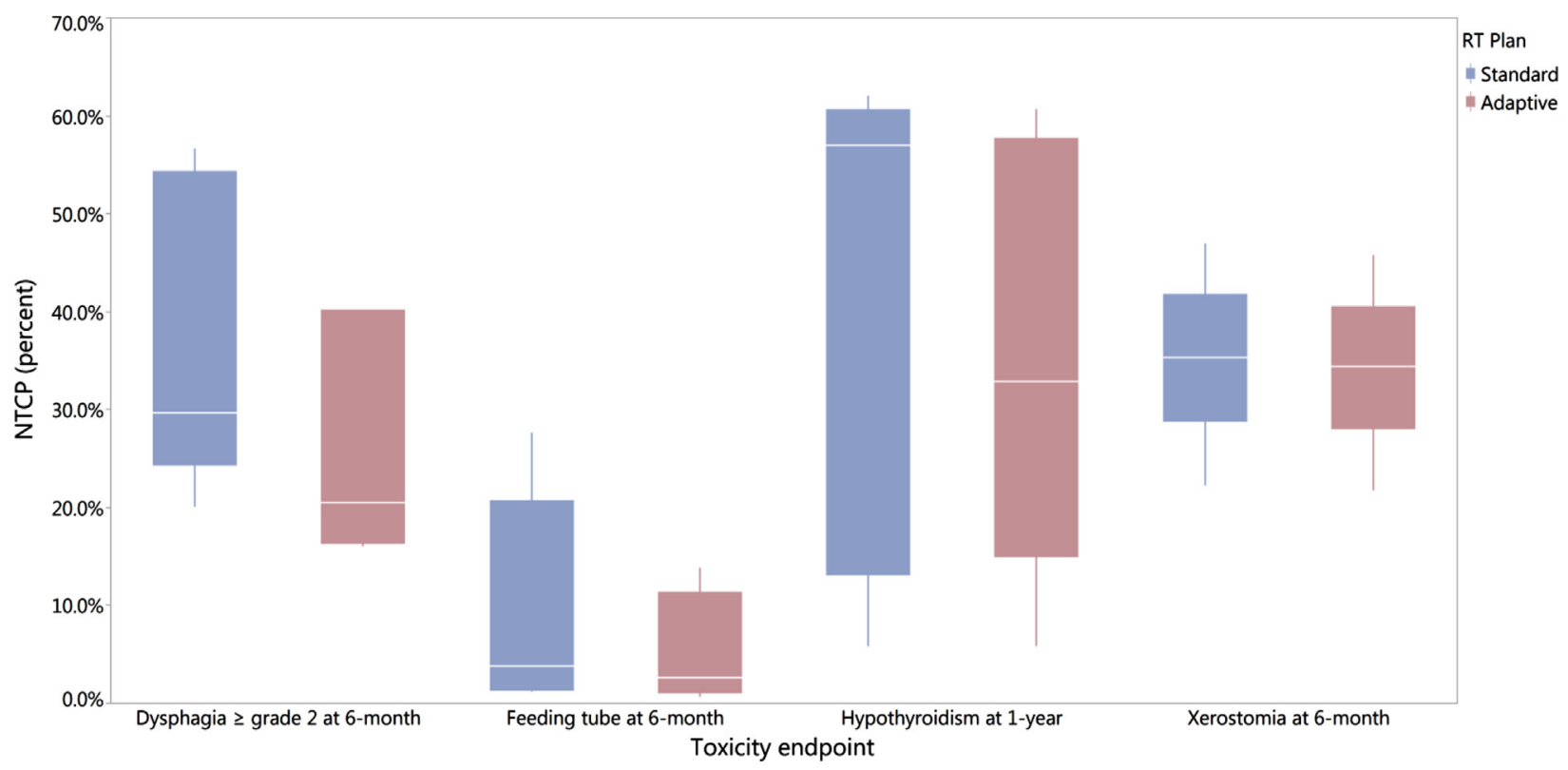

Fig. 4. Boxplots depicting the comparison of normal tissue complication probability (NTCP) between standard and adaptive methods.

the first year after randomization to either IMRT or TORS in OPC patients.

It is fairly well established that radiation dose is closely related to radiation-induced long-term toxicities, notably to rates and severity of dysphagia as well as rates of stricture formation, feeding tube dependence and aspiration [39-41]. In a recent systematic review by Duprez et al., mean dose to pharyngeal constrictors was the strongest predictor of late swallowing dysfunction, with clinical reduction of swallowing dysfunction observed with dose of 5255 Gy vs. 61-64 Gy, suggesting that even mean OAR dose reduction of less than $10 \mathrm{~Gy}$ could translate into clinically impactful toxicity reduction [42]. In this context, de-escalation strategies aiming at reducing radiation dose are particularly appealing. Chera et al. [35] recently investigated rates of complete response of a deintensified chemoradiation strategy in favorable risk HPV+ OPC. Treatment de-escalation consisted of delivery of $60 \mathrm{~Gy}$ to the gross disease and reduced cisplatin dose $\left(30 \mathrm{mg} / \mathrm{m}^{2}\right.$ weekly). The reported clinical complete response rates reached $98 \%$ and $60 \%$ at the primary and regional sites respectively, suggesting that dose de-escalation may be suitable in selected patients. However, the optimal strategy for patients' selection, notably the potential role of adaptive de-escalation based on individual response, remains to be investigated.

The principle of adaptive radiotherapy planning relies on monitoring temporal and spatial anatomical changes over the course of radiotherapy, and modulating radiation dose based on observed changes. These changes can include changes in target volumes, OAR volume or shape, weight loss, alteration in muscle mass, or edema $[13,43,44]$. Several previous studies have assessed the role of per-treatment imaging response during the course of radiotherapy for head and neck cancer, including CT [13,14,45,46], PET-CT [47], anatomic MRI and functional MRI (diffusion weighted or dynamic contrast enhanced) [48], with tumor changes observed in the majority of patients, as early as by fraction 11 [49]. Using CT-on-rails image guidance in patients undergoing head and neck radiotherapy, Schwartz et al. at reported that all patients benefited from at least one re-plan and 36\% required a second re-plan to account for weight loss, CTV and normal tissue changes $[13,14]$. More recently, Lee et al. [36] reported outcomes of an adaptive approach consisting of $10 \mathrm{~Gy}$ dose de-escalation to involved lymph nodes based on early treatment hypoxia assessment using 18F-
fluoromisonidazole-PET. Among 33 patients, 30\% received reduced radiation dose; 2-year locoregional control rate was as high as $100 \%$. This study suggests that functional imaging may play an important role in guiding adaptive radiation strategies. The increasing use of MRI for head and neck radiotherapy planning has the advantage of improved soft-tissue visualization [15], which allows to more confidently assess anatomical tumor changes during treatment. In addition, MRI also offers the possibility of frequent per-treatment functional assessment, without the addition of ionizing radiation. The recent introduction of the MR-Linac technology holds the promise to facilitate such adaptive IMRT workflows by mean of daily on-line MRI during radiation treatment [50].

This in silico study is limited by its small sample size. However, the aim of this study was to establish the feasibility and the dosimetric advantage of this proposed MRI-guided IMRT dose-adaption workflow for HPV+ OPC, in preparation for future clinical application. In addition, this study used only anatomical MR-sequences for treatment adaption. However, although the role of functional MRI certainly seems promising for assessment and prediction of tumor response [48], observed functional changes require further investigation to establish clear thresholds to be used clinically for treatment adaptation. Finally, the safety, in terms of cancer control outcomes, as well as the toxicity advantages of this workflow will be validated in an upcoming clinical trial by our institution. The results of this study guided the sample size calculation of this upcoming phase II clinical trial designed to validate the superiority of MRI-guided radiotherapy dose adaptation for improving the toxicity profile of HPV+ oropharyngeal cancers without compromising the outcomes.

\section{Conclusion}

This in silico results showed the suggested MRI-guided adaptive approach is technically feasible, safe (with no normal tissue exceeding modeled dose constraints), and advantageous in reducing dose to OARs, especially swallowing musculature, thus reducing the NTCP of dysphagia $\geq$ grade 2 , feeding tube persistence at 6-month post-treatment, and hypothyroidism at 1-year posttreatment. 


\section{Conflict of interest statement}

The authors declare no conflicts of interest.

\section{Funding sources and financial disclosures}

Drs. Mohamed, Lai, Hutcheson, and Fuller receive(d) funding support from the National Institutes of Health (NIH)/National Institute for Dental and Craniofacial Research (1R01DE025248-01/R56 DE025248-01) and NIH/NCI Early Phase Clinical Trials in Imaging and Image-Guided Interventions Program (1R01CA218148-01). Dr. Fuller received/receives federal grant and/or salary support from: the NIH/National Cancer Institute (NCI) Head and Neck Specialized Programs of Research Excellence (SPORE) Developmental Research Program Award (P50CA097007-10) and Pau 1 Calabresi Clinical Oncology Program Award (K12 CA088084-06); a National Science Foundation (NSF), Division of Mathematical Sciences, Joint NIH/NSF Initiative on Quantitative Approaches to Biomedical Big Data (QuBBD) Grant (NSF 1557679); the NIH Big Data to Knowledge (BD2K) Program of the National Cancer Institute (NCI) Early Stage Development of Technologies in Biomedical Computing, Informatics, and Big Data Science Award (1R01CA214825-01); and the Cancer center Support Grant Radiation Oncology/Cancer Imaging Program Seed Grant (5P30CA016672). Dr. Fuller receives (d) industry grant support and speaker travel funding from Elekta AB. Dr. Fuller is a Sabin Family Foundation Fellow. Dr. Rosenthal receives grants from: $\mathrm{NIH} / \mathrm{NCI}$ (5R0CA148707-03) and (5R01CA160880-03).

\section{Appendix A. Supplementary data}

Supplementary data associated with this article can be found, in the online version, at https://doi.org/10.1016/j.ctro.2018.04.005.

\section{References}

[1] Gillison ML, Chaturvedi AK, Anderson WF, Fakhry C. Epidemiology of human papillomavirus-positive head and neck squamous cell carcinoma. J Clin Oncol 2015;33(29):3235-42

[2] Garden AS, Kies MS, Morrison WH, Weber RS, Frank SJ, Glisson BS, et al Outcomes and patterns of care of patients with locally advanced oropharyngeal carcinoma treated in the early 21(st) century. Radiat Oncol (London, England) 2013;8:21.

[3] Haughey BH, Sinha P. Prognostic factors and survival unique to surgically treated p16+ oropharyngeal cancer. Laryngoscope 2012;122(S2):S13-33.

[4] Licitra L, Perrone F, Bossi P, Suardi S, Mariani L, Artusi R, et al. High-risk human papillomavirus affects prognosis in patients with surgically treated oropharyngeal squamous cell carcinoma. J Clin Oncol 2006;24(36):5630-6.

[5] Mendenhall WM, Amdur RJ, Morris CG, Kirwan JM, Li JG. Intensity-modulated radiotherapy for oropharyngeal squamous cell carcinoma1. Laryngoscope 2010;120(11):2218-22.

[6] Sedaghat AR, Zhang Z, Begum S, Palermo R, Best S, Ulmer KM, et al. Prognostic significance of human papillomavirus in oropharyngeal squamous cell carcinomas. Laryngoscope 2009;119(8):1542-9.

[7] Toledano I, Graff P, Serre A, Boisselier P, Bensadoun R-J, Ortholan C, et al. Intensity-modulated radiotherapy in head and neck cancer: results of the prospective study GORTEC 2004-03. Radiother Oncol 2012;103(1):57-62.

[8] Ang KK, Harris J, Wheeler R, Weber R, Rosenthal DI, Nguyen-Tân PF, et al. Human papillomavirus and survival of patients with oropharyngeal cancer. $\mathrm{N}$ Engl J Med 2010;363(1):24-35.

[9] Eisbruch A, Schwartz M, Rasch C, Vineberg K, Damen E, Van As CJ, et al Dysphagia and aspiration after chemoradiotherapy for head-and-neck cancer: which anatomic structures are affected and can they be spared by IMRT? Int Radiat Oncol Biol Phys 2004;60(5):1425-39.

[10] Levendag PC, Teguh DN, Voet P, van der Est H, Noever I, de Kruijf WJM, et al. Dysphagia disorders in patients with cancer of the oropharynx are significantly affected by the radiation therapy dose to the superior and middle constrictor muscle: a dose-effect relationship. Radiother Oncol 2007;85(1):64-73.

[11] Mortensen HR, Jensen K, Aksglæde K, Behrens M, Grau C. Late dysphagia after IMRT for head and neck cancer and correlation with dose-volume parameters. Radiother Oncol 2013;107(3):288-94
[12] Wilson JA, Carding PN, Patterson JM. Dysphagia after nonsurgical head and neck cancer treatment: patients' perspectives. Otolaryngol Head Neck Surg 2011;145(5):767-71.

[13] Schwartz DL, Garden AS, Shah SJ, Chronowski G, Sejpal S, Rosenthal DI, et al. Adaptive radiotherapy for head and neck cancer-dosimetric results from a prospective clinical trial. Radiother Oncol 2013;106(1):80-4.

[14] Schwartz DL, Garden AS, Thomas J, Chen Y, Zhang Y, Lewin J, et al. Adaptive radiotherapy for head-and-neck cancer: initial clinical outcomes from a prospective trial. Int J Radiat Oncol Biol Phys 2012;83(3):986-93.

[15] Wippold 2nd FJ. Head and neck imaging: the role of CT and MRI. JMRI 2007;25 (3):453-65.

[16] Ding Y, Hazle JD, Mohamed AS, Frank SJ, Hobbs BP, Colen RR, et al. Intravoxel incoherent motion imaging kinetics during chemoradiotherapy for human papillomavirus-associated squamous cell carcinoma of the oropharynx: preliminary results from a prospective pilot study. NMR Biomed 2015;28 (12):1645-54.

[17] Ding Y, Mohamed ASR, Yang J, Colen RR, Frank SJ, Wang J, et al. Prospective observer and software-based assessment of magnetic resonance imaging quality in head and neck cancer: should standard positioning and immobilization be required for radiation therapy applications? Pract Radiat Oncol 2015;5(4):e299-308.

[18] Cardenas CE, Mohamed ASR, Tao R, Wong AJR, Awan MJ, Kuruvila S, et al. Prospective qualitative and quantitative analysis of real-time peer review quality assurance rounds incorporating direct physical examination for head and neck cancer radiation therapy. Int J Radiat Oncol Biol Phys 2017;98 (3):532-40.

[19] Wopken K, Bijl HP, van der Schaaf A, van der Laan HP, Chouvalova O, Steenbakkers RJHM, et al. Development of a multivariable normal tissue complication probability (NTCP) model for tube feeding dependence after curative radiotherapy/chemo-radiotherapy in head and neck cancer. Radiother Oncol 2014;113(1):95-101.

[20] Christianen MEMC, Schilstra C, Beetz I, Muijs CT, Chouvalova O, Burlage FR, et al. Predictive modelling for swallowing dysfunction after primary (chemo) radiation: results of a prospective observational study. Radiother Oncol 2012;105(1):107-14.

[21] Boomsma MJ, Bijl HP, Christianen MEMC, Beetz I, Chouvalova O, Steenbakkers RJHM, et al. A prospective cohort study on radiation-induced hypothyroidism: development of an NTCP model. Int J Radiat Oncol Biol Phys 2012;84(3): e351-6.

[22] Beetz I, Schilstra C, van der Schaaf A, van den Heuvel ER, Doornaert P, van Luijk $P$, et al. NTCP models for patient-rated xerostomia and sticky saliva after treatment with intensity modulated radiotherapy for head and neck cancer: the role of dosimetric and clinical factors. Radiother Oncol 2012;105(1):101-6.

[23] Blanchard P, Wong AJ, Gunn GB, Garden AS, Mohamed ASR, Rosenthal DI, et al. Toward a model-based patient selection strategy for proton therapy: External validation of photon-derived normal tissue complication probability models in a head and neck proton therapy cohort. Radiother Oncol 2016;121(3):381-6.

[24] Ang KK, Sturgis EM. Human papillomavirus as a marker of the natural history and response to therapy of head and neck squamous cell carcinoma. Semin Radiat Oncol 2012;22(2):128-42.

[25] Ang KK, Harris J, Wheeler R, Weber R, Rosenthal DI, Nguyen-Tan PF, et al. Human papillomavirus and survival of patients with oropharyngeal cancer. $\mathrm{N}$ Engl J Med 2010;363(1):24-35.

[26] Jung AC, Briolat J, Millon R, de Reynies A, Rickman D, Thomas E, et al. Biological and clinical relevance of transcriptionally active human papillomavirus (HPV) infection in oropharynx squamous cell carcinoma. Int J Cancer 2010;126 (8):1882-94

[27] Doescher J, Veit JA, Hoffmann TK. The 8th edition of the AJCC Cancer Staging Manual: updates in otorhinolaryngology, head and neck surgery. Hno 2017.

[28] Langendijk JA, Doornaert P, Verdonck-de Leeuw IM, Leemans CR, Aaronson NK, Slotman BJ. Impact of late treatment-related toxicity on quality of life among patients with head and neck cancer treated with radiotherapy. J Clin Oncol 2008;26(22):3770-6.

[29] Kofler B, Laban S, Busch CJ, Lorincz B, Knecht R. New treatment strategies for HPV-positive head and neck cancer. Eur Arch Otorhinolaryngol 2014;271 (7):1861-7.

[30] Masterson L, Moualed D, Liu ZW, Howard JE, Dwivedi RC, Tysome JR, et al. Deescalation treatment protocols for human papillomavirus-associated oropharyngeal squamous cell carcinoma: a systematic review and metaanalysis of current clinical trials. Eur J Cancer (Oxford, England: 1990) 2014;50 (15):2636-48

[31] Mirghani H, Amen F, Blanchard P, Moreau F, Guigay J, Hartl DM, et al. Treatment de-escalation in HPV-positive oropharyngeal carcinoma: ongoing trials, critical issues and perspectives. Int J Cancer 2015;136(7):1494-503.

[32] Marur S, Li S, Cmelak AJ, Gillison ML, Zhao WJ, Ferris RL, et al. E1308: Phase II trial of induction chemotherapy followed by reduced-dose radiation and weekly cetuximab in patients with HPV-associated resectable squamous cell carcinoma of the oropharynx-ECOG-ACRIN Cancer Research Group. J Clin Oncol 2017;35(5):490-7.

[33] Chen AM, Felix C, Wang PC, Hsu S, Basehart V, Garst J, et al. Reduced-dose radiotherapy for human papillomavirus-associated squamous-cell carcinoma of the oropharynx: a single-arm, phase 2 study. Lancet Oncol 2017;18 (6):803-11.

[34] Rainey H, Roy E, Selkridge I. Abstract (Standard of care vs reduced-dose chemoradiation after induction chemotherapy in HPV + oropharyngeal carcinoma patients). J Clin Oncol 2017;35(1):6069. 
[35] Chera BS, Amdur RJ, Tepper J, Qaqish B, Green R, Aumer SL, et al. Phase 2 trial of de-intensified chemoradiation therapy for favorable-risk human papillomavirus-associated oropharyngeal squamous cell carcinoma. Int J Radiat Oncol Biol Phys 2015;93(5):976-85.

[36] Lee N, Schoder H, Beattie B, Lanning R, Riaz N, McBride S, et al. Strategy of using intratreatment hypoxia imaging to selectively and safely guide radiation dose de-escalation concurrent with chemotherapy for locoregionally advanced human papillomavirus-related oropharyngeal carcinoma. Int J Radiat Oncol Biol Phys 2016;96(1):9-17.

[37] Blanchard P, Wong AJ, Gunn GB, Garden AS, Mohamed ASR, Rosenthal DI, et al. Toward a model-based patient selection strategy for proton therapy: External validation of photon-derived normal tissue complication probability models in a head and neck proton therapy cohort. Radiother Oncol 2016;121(3):381-6.

[38] Dowthwaite SA, Franklin JH, Palma DA, Fung K, Yoo J, Nichols AC. The role of transoral robotic surgery in the management of oropharyngeal cancer: a review of the literature. ISRN Oncol 2012;2012:945162.

[39] Cartmill B, Cornwell P, Ward E, Davidson W, Nund R, Bettington C, et al. Emerging understanding of dosimetric factors impacting on dysphagia and nutrition following radiotherapy for oropharyngeal cancer. Head Neck 2013;35(8):1211-9.

[40] Feng FY, Kim HM, Lyden TH, Haxer MJ, Feng M, Worden FP, et al. Intensitymodulated radiotherapy of head and neck cancer aiming to reduce dysphagia: early dose-effect relationships for the swallowing structures. Int J Radiat Oncol Biol Phys 2007;68(5):1289-98.

[41] Caudell JJ, Schaner PE, Desmond RA, Meredith RF, Spencer SA, Bonner JA. Dosimetric factors associated with long-term dysphagia after definitive radiotherapy for squamous cell carcinoma of the head and neck. Int J Radiat Oncol Biol Phys 2010;76(2):403-9.

[42] Duprez F, Madani I, De Potter B, Boterberg T, De Neve W. Systematic review of dose-volume correlates for structures related to late swallowing disturbances after radiotherapy for head and neck cancer. Dysphagia 2013;28(3):337-49.
[43] Barker Jr JL, Garden AS, Ang KK, O'Daniel JC, Wang H, Court LE, et al Quantification of volumetric and geometric changes occurring during fractionated radiotherapy for head-and-neck cancer using an integrated CT/linear accelerator system. Int J Radiat Oncol Biol Phys 2004;59(4):960-70.

[44] Hansen EK, Bucci MK, Quivey JM, Weinberg V, Xia P. Repeat CT imaging and replanning during the course of IMRT for head-and-neck cancer. Int J Radiat Oncol Biol Phys 2006;64(2):355-62.

[45] Geets X, Tomsej M, Lee JA, Duprez T, Coche E, Cosnard G, et al. Adaptive biological image-guided IMRT with anatomic and functional imaging in pharyngo-laryngeal tumors: impact on target volume delineation and dose distribution using helical tomotherapy. Radiother Oncol 2007;85(1):105-15.

[46] Veiga C, McClelland J, Moinuddin S, Lourenco A, Ricketts K, Annkah J, et al. Toward adaptive radiotherapy for head and neck patients: feasibility study on using CT-to-СBCT deformable registration for "dose of the day" calculations. Med Phys 2014;41(3):031703.

[47] Bussink J, van Herpen CM, Kaanders JH, Oyen WJ. PET-CT for response assessment and treatment adaptation in head and neck cancer. Lancet Oncol 2010;11(7):661-9.

[48] Dirix P, Vandecaveye V, De Keyzer F, Stroobants S, Hermans R, Nuyts S. Dose painting in radiotherapy for head and neck squamous cell carcinoma: value of repeated functional imaging with (18)F-FDG PET, (18)F-fluoromisonidazole PET, diffusion-weighted MRI, and dynamic contrast-enhanced MRI. J Nucl Med 2009;50(7):1020-7.

[49] Subesinghe M, Scarsbrook AF, Sourbron S, Wilson DJ, McDermott G, Speight R, et al. Alterations in anatomic and functional imaging parameters with repeated FDG PET-CT and MRI during radiotherapy for head and neck cancer: a pilot study. BMC Cancer 2015;15:137.

[50] Kontaxis C, Bol GH, Lagendijk JJ, Raaymakers BW. Towards adaptive IMRT sequencing for the MR-linac. Phys Med Biol 2015;60(6):2493-509. 